\title{
Fusion of local and global features for classification of abnormality in mammograms
}

\author{
ANURADHA C PHADKE** and PRITI P REGE \\ Department of Electronics and Telecommunication, College of Engineering, Pune 411005, India \\ e-mail: anuradha.phadke@mitpune.edu.in; ppr.extc@coep.ac.in
}

MS received 18 April 2015; revised 4 August 2015; accepted 17 November 2015

\begin{abstract}
Mammography is the most widely used tool for the early detection of breast cancer. Computerbased algorithms can be developed to improve diagnostic information in mammograms and assist the radiologist to improve diagnostic accuracy. In this paper, we propose a novel computer aided technique to classify abnormalities in mammograms using fusion of local and global features. The objective of this work is to test the effectiveness of combined use of local and global features in detecting abnormalities in mammograms. Local features used in the system are Chebyshev moments and Haralick's gray level co-occurrence matrix based texture features. Global features used are Laws texture energy measures, Gabor based texture energy measures and fractal dimension. All types of abnormalities namely clusters of microcalcifications, circumscribed masses, spiculated masses, architectural distortions and ill-defined masses are considered. A support vector machine classifier is designed to classify the samples into abnormal and normal classes. It is observed that combined use of local and global features has improved classification accuracy from $88.75 \%$ to $93.17 \%$.
\end{abstract}

Keywords. Mammogram; Laws texture features; Gabor transform; fractal dimension; SVM; GLCM.

\section{Introduction}

Breast cancer has been identified as the most widespread cancer amongst women and also the major cause of female cancer death all over the world. In India, breast cancer is widespread in urban areas as well as rural areas. Over the last few decades, the average age of developing a breast cancer is reducing significantly. In the year 2008, there were about 115,251 new breast cancer cases diagnosed in India [1]. It is estimated that by 2030 , the number of breast cancer in India will be 200,000 per year. As the cause of developing breast cancer is not yet completely understood, at present there are no effective ways to prevent breast cancer. Early detection of breast cancer and treatment during the initial stages increases the possibility of full recovery. Mammography is the most commonly used technique for early detection of breast cancer, in which the disease is detected at a pre-symptomatic phase. With the aid of mammogram, it is possible to capture and analyze inner texture of breast. Computer assisted diagnosis (CAD) of mammograms attempts to help radiologists providing an automatic procedure to detect possible cancers in mammograms.

When the natural regulators do not work, breast cells begin to multiply and spread. This activity results in a tumor. Most of the breast cancers begin in the milk ducts,

*For correspondence and a few begin in the lobules or other breast tissues. When abnormal cells grow inside the ducts but do not spread to the nearby tissue and other body parts, it is known as benign abnormality. A benign tumor is not dangerous to health and hence not considered as cancerous. Benign tumor grows slowly and its appearance is very close to normal cells. In case of malignant tumors abnormal growth invades the tissue and may spread to the other body parts. Hence these tumors are more serious and known as cancer. Risk can be reduced if malignant tumors are detected and treated in time.

Radiologists visually examine mammograms for specific abnormalities. Some of the important signs of breast cancer that radiologists look for are clusters of microcalcifications (CALC), circumscribed masses (MASS), spiculated masses (SPIC), architectural distortions (ARCH) and ill-defined or miscellaneous masses (MISC). Breast lesions are described and reported according to the breast imaging reporting and data system (BI-RADS) given by American College of Radiology [2]. The BI-RADS, developed by the American College of Radiology, provides a standardized classification for mammographic studies [3]. Figure 1 shows examples of five types of abnormalities and normal region of interest (ROI). In mammogram images, masses are described by their shape and margin characteristics. Calcifications are tiny deposits of calcium, which appear as small bright spots on the mammogram. They are characterized by their type and distribution properties. Distortion in the normal breast 


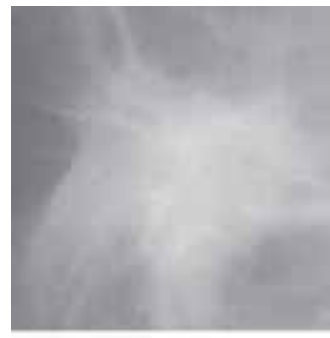

(a)

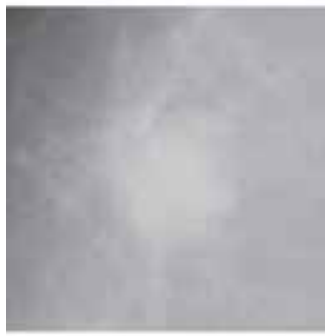

(d)

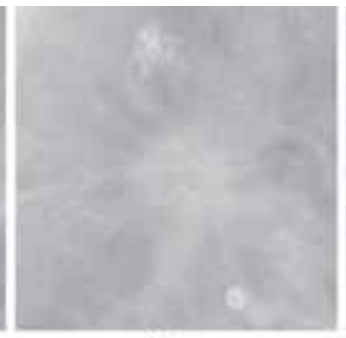

(b)

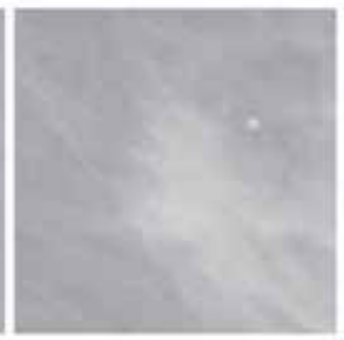

(e)

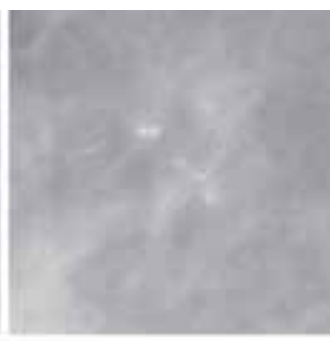

(c)

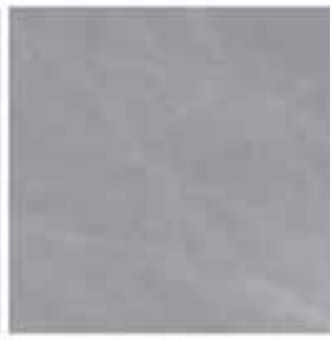

(f)

Figure 1. (a) Architectural distortion; (b) spiculated mass; (c) microcalcification; (d) circumscribed mass; (e) miscellaneous; (f) normal.

architecture is an indication of abnormality and it is known as architectural distortion. Unlike circumscribed masses, there is no definite visible mass. It may include distortion of the edge of the parenchyma, focal retraction or speculation radiating from a point.

Detection of suspicious abnormalities is a repetitive and fatiguing task. Because of the large number of normal patients in the screening programs, there is a risk that radiologists may miss some subtle abnormalities, which may account to failure. CAD provides a second opinion to the radiologist and helps them in increasing the accuracy of detection. Developing CAD algorithms is an extremely exigent task. Imperfections in the mammogram capturing system, large variability in the appearance of abnormal regions, variability in the background tissue structure of breast, hidden abnormality in dense breast tissues, irregular textures with subtle similarities and differences are some challenges which are to be considered while designing the system. One cannot deal with such type of image analysis problem by looking for a well-behaved visual template or structural data.

In this paper, we propose a method to classify given ROI in mammogram as normal or abnormal. The similarity in intensities with the normal tissue and similarities in morphology with other normal textures in the breast makes it more difficult to detect abnormalities. A review of existing techniques for mammographic feature analysis and detection of abnormalities is presented. Vibha \& Rege [4] proposed use of Chebyshev moments for identification of malignant mammogram and the type of abnormality. In this work they have not mentioned any performance measures. Rashed Essam et al [5] presented a multiresolution analysis system using DB (Daubechies) wavelets. Given ROI is decomposed up to fourth level using DB wavelet and from LL component of each level, fractional amount of biggest wavelet coefficients are selected to form feature vector. Classification is performed using Euclidian distance based classifier. It is observed that DB8 wavelet gives highest classification accuracy. Tirtajaya Andy \& Santika Diaz [6] implemented a system where features are extracted using dual-tree complex wavelet transform technique and classification of benign and malignant lesions is performed using support vector machine (SVM). The method produced good classification accuracy of $88.64 \%$. Work by Nithya \& Santhi [7] is based on gray level co-occurrence matrix features. The relevant features for classification of mammograms are selected using maximum difference feature selection method and a neural network classifier is trained with the selected features. It is reported that classification accuracy is improved because of the proposed feature selection method. The framework by Phadke \& Rege [8] is based on combining principal component analysis (PCA) and independent component analysis (ICA) for deriving features of normal and malignant tissues in mammograms. Classification of malignant tissues is done by SVM and ANN Classifier and their performance is compared. It is observed that ICA features are effective in classifying malignant tissues from normal tissues. Shanthi \& Murali Bhaskaran [9] proposed a system to detect lesions in mammograms using features based on Gabor filters, fractal analysis and multi-scale surrounding region dependence method. Proposed features with self-adaptive resource allocation network (SRAN) classifier improved the classification performance. From the experimentation, it was concluded that proposed features with SRAN classifier improved the classification performance. 
Ted C Wang \& Nicolaos B Karayiannis [10] detected microcalcifications by decomposing the mammograms into different frequency sub-bands. After decomposition, lowfrequency sub-band is suppressed and finally, the mammogram is reconstructed from high frequency sub-bands only. In this work some basic experimentation of use of wavelet decomposition for the detection of microcalcification is reported. It suggests further studies in this domain for checking suitability of wavelet decomposition for detection of microcalcifications. In the work by Karahaliou et al [11] ROI containing the microcalcifications was preprocessed using a wavelet based contrast enhancement method, followed by local thresholding to segment microcalcifications. Various texture features are extracted from the tissues surrounding the microcalcifications. A k-nearest neighbor classifier is used to differentiate between malignant and benign microcalcifications. Highest accuracy of $89 \%$ is achieved with the use of Laws' texture energy measures. Alarcon-Aquino et al [12] proposed an approach to detect microcalcifications in digital mammograms using the dual-tree complex wavelet transform (DT-CWT). The approach follows four basic strategies, namely, image denoising, band suppression, morphological transformation and inverse complex wavelet transform. The combination of morphological transformation and DT-CWT performs better than the stationary and discrete wavelet transforms and the top-hat filtering. Balakumaran et al [13] have proposed an algorithm for detecting microcalcification in mammogram. The proposed microcalcification detection algorithm involves mammogram quality enhancement using multiresolution analysis based on the dyadic wavelet transform and microcalcification detection by fuzzy shell clustering. Limitation of this method is that it can detect only nodular microcalcifications. Leena et al [14] used combination of non sub sampled contourlet transform (NSCT) and artificial neural networks (ANN) for detecting microcalcification in digital mammograms and achieved classification rate of $83.5 \%$. The framework by Paradkar \& Pande [15] uses back propagation neural network classifier for detection of microcalcification from digital mammograms. The number of candidate pixels is reduced by neuron-based thresholding strategy. They have suggested use of connected component analysis followed by the elongated component removal algorithms to eliminate the false positives. The system was found to be robust, accurate and efficient. Oliver et al [16] proposed a knowledge-based system for detecting microcalcifications and clusters in mammograms automatically. Local description of the microcalcifications morphology is obtained by using bank of filters. A set of extracted features is used to learn the classifier. Proposed system gives sensitivity of $80 \%$ at one false positive per image.

Sampat \& Bovik [17] present a system where enhancement of spiculation is done using Radon transform and column filter to detect 'low high low' patterns and detection of spiculations is performed by using sine and cosine radial speculation filter. Preliminary experimentation for detection of speculated lesion is done only on few mammograms. Biswas \& Dipti [18] proposed a system with two layer architecture for detection of architectural distortion in mammograms. In the first layer, low level rotation invariant, textural features at different scales are obtained by filtering the ROI using oriented filter bank. In the second layer, learning of fundamental patterns i.e. textons is done from observed texture descriptors. The set of textons is generated using Gaussian mixture model. This technique reported accuracy of $81.6 \%$. Banik et al [19] made use of Gabor filters, analysis of phase map and angular spread of power, fractal and texture analysis for the detection of architectural distortion in mammograms of interval cancer cases. Limitation of this technique is that it can detect only node like speculated patterns of the architectural distortion. Phadke \& Rege [20] applied Gabor filters and used Laws texture features of Gabor filtered image to detect architectural distortion. In this method comparatively smaller database is used and accuracy of $82.86 \%$ is achieved.

Rangayyan \& Nguyen [21] worked on the application of fractal analysis to breast masses. Here, the fractal dimensions of the breast mass contours were computed using the ruler and box counting methods. The results show that there is significant difference between fractal dimension of benign and malignant masses. Apffel Louis et al [22] presented a fuzzy segmentation method to segment masses in digital breast tomosynthesis datasets by deriving the optimal contour, i.e. the path associated with a minimal cost. Several relevant contours from a single image are extracted by altering the cost by several ways. Lesion is detected by using the set of contours with the fuzzy contour segmentation method. The method is evaluated only by visual approach as creating a ground truth for fuzzy contours is a very difficult task.

Arianna Mencattini et al [23] carried a work on breast mass segmentation in mammographic images by spatial fuzzy C-means segmentation for boundary detection followed by boundary refinement using active contour models. The algorithm works well on direct digital mammograms as well as digitized mammograms.

The abnormalities like microcalcifications, circumscribed masses can be differentiated well from normal tissues by the texture of small region around the center of abnormality. Texture features over a local neighborhood help in identifying these types of abnormalities. Gray Level Co-occurrence Matrix based texture features and Chebyshev moments over a small area around the center of the ROI are effective in detecting abnormality types like masses and microcalcifications. Architectural distortion and large speculated lesions are usually of greater size and show features like oriented patterns. In order to detect these abnormalities global neighborhood of size $128 \times 128$ with Gabor features and Laws' texture measures are used [19]. If only local features are used, architectural distortion and speculated lesions might go undetected. If only global 
features are used microcalcifications might be missed. Thus combined use of local and global features is essential. In this work, we have used combination of these methods of feature extraction.

The paper is organized as follows: Section 2 gives details of database used in the study and generation of sub-images. Section 3 covers the proposed method with block schematic, feature extraction methods, and SVM classifier. Section 4 highlights the simulation results and discussions. Finally, conclusions are given in Section 5 .

\section{Database and sub-image generation}

The mammogram images used in this work are taken from the mini mammography database of MIAS, the Mammographic Image Analysis Society [24]. The images in the MIAS are captured in Medeo-lateral Oblique view (MLO). In the mini mammography database, original 50 micron pixel edge resolution has been reduced to 200 micron pixel edge and by clipped/padded every image is converted to 1024 pixels $\times 1024$ pixels. All images are held as 8-bit gray level scale images with 256 different gray levels (0-255).

Since MIAS images are very large in size, ROI is selected by cropping the images. MIAS database provides center and radius of abnormality for all the images. The images are cropped to size $128 \times 128$ squared around the given center. MIAS database provides total 322 mammograms. It consists of 207 normal, 63 benign and 52 malignant cases. It includes all types of abnormalities like microcalcifications, circumscribed masses, spiculated masses, architectural distortions, asymmetry and miscellaneous abnormality. Except asymmetry, all other types of abnormalities are considered in this work. Total 96 abnormal ROIs and 153 normal ROIs are generated and used in implementing the system. Abnormal ROI database is composed of 42 malignant and 54 benign types of abnormalities.

\section{Methods}

Primary focus of this work is to develop a system to classify abnormality from normal samples at a very early stage. Figure 2 demonstrates the methodology adopted for the design of the system.

The proposed system extracts local and global features from the given region of interest. A small neighborhood of size less than or equal to $32 \times 32$ is used to extract texture features in the local area of abnormality. Local features used in the system are Chebyshev moments and 19 gray level co-occurrence matrix based texture features. Global features used are Laws texture energy measures, Gabor based texture energy measures and fractal dimension.
Global features characterize the global texture of the ROI of size $128 \times 128$. Local and global features together describe the texture of given ROI. Further the extracted features are used to train the SVM classifier for ROI classification.

\subsection{Pre-processing}

Pre-processing of ROI is needed in order to enhance the features of interest. Pre-processing technique to be applied is selected on the basis of feature to be extracted.

- Pre-processing for local features: Computation of the Chebyshev moments is preceded by median filtering of the ROI. Median filter smoothes out the high frequency data which is not of interest. Mammograms usually possess low contrast and hence prior to the computation of GLCM features, contrast of the ROI is enhanced by applying contrast limited adaptive histogram equalization.

- Pre-processing for global features: Unsharp masking is used as preprocessing step before extracting Gabor filtered based global features. Given ROI is first filtered using Gaussian smoothing filter with kernel given as

$$
s(x, y)=e^{-a} \text { with } a=\frac{\left(x^{2}+y^{2}\right)}{2 . r^{2}},
$$

where $x$ and $y$ are spatial coordinates of the pixels in the ROI and $r$ is the filter radius. The filter radius $r$ is the standard deviation used to filter the ROI and is taken as 20. Gaussian kernel $s(x, y)$ is convolved with ROI $f(x, y)$ to get blurred version of image $b(x, y)$ which is subtracted from original ROI $f(x, y)$ to get the sharpened image $h(x, y)$.

$$
h(x, y)=f(x, y)-b(x, y) .
$$

Because of sharpening operation, the features of interest like node patterns in architectural distortion, radiating patterns in spiculations, bright spots in microcalcifications are enhanced. An example of abnormal ROI and the results of applying Gaussian filter and unsharp masking on the abnormal ROI are given in figure 3(a), (b) and (c) respectively.

\subsection{Local feature extraction}

Several pattern recognition applications use Haralick's GLCM texture features as it characterizes the spatial distribution of gray levels in the image. Discrete orthogonal moments are also used to capture image features such as shape and texture. In the proposed work, features over a small neighborhood around the center of the ROI are captured in terms of 19 GLCM features and two discrete moments such as Chebyshev moments. Local feature extraction methodology is demonstrated in figure 4 . 


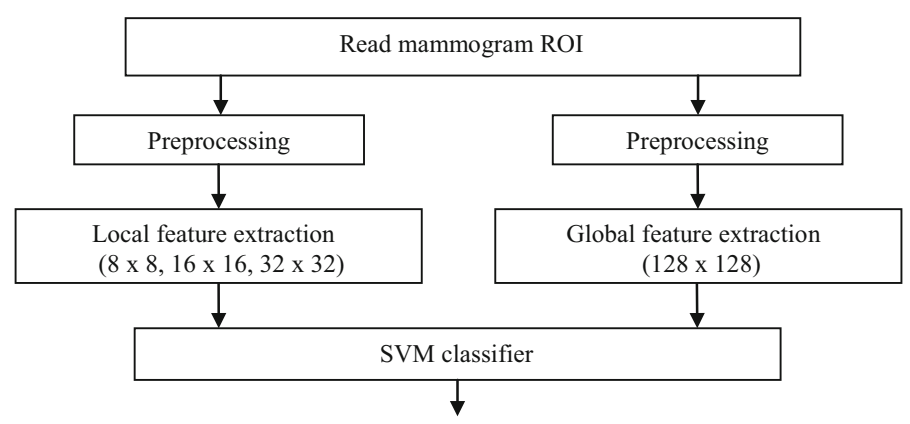

Figure 2. Steps for classifying abnormal ROIs in the sample database.

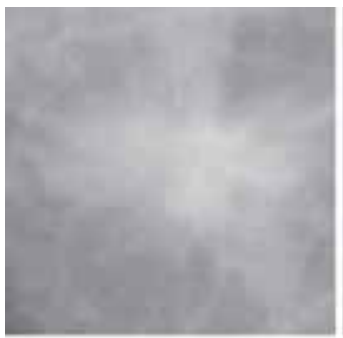

(a)

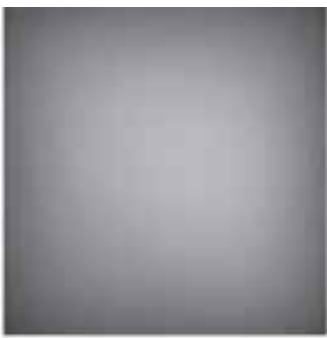

(b)

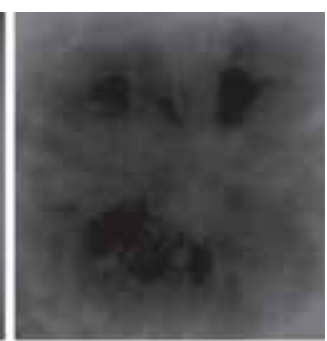

(c)

Figure 3. (a) Abnormal ROI; (b) Gaussian filtered ROI; (c) sharpened ROI.

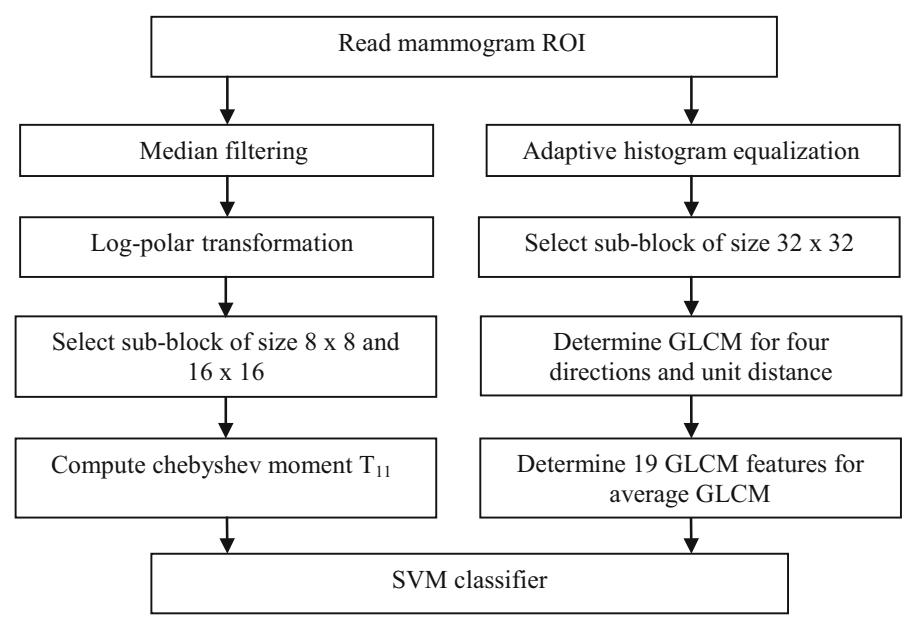

Figure 4. Local feature extraction for ROI.

3.2a GLCM features: GLCM is a robust statistical technique used to estimate second order statistics. Each element in the GLCM represents probability of occurrence of the pair of gray levels [25]. First step is to enhance the contrast of the ROI using adaptive histogram equalization. Then a sub-block of size $32 \times 32$ at the center of ROI is selected and its pixel values are quantized to 32 levels from original 256 levels and GLCMs are determined for unit distance and four directions. Average of these GLCMs is used to determine 19 features namely autocorrelation, contrast, correlation, cluster prominence, cluster shade, dissimilarity, energy, entropy, homogeneity, maximum probability, sum of squares variance, sum average, sum variance, sum entropy, difference variance, difference entropy, information measure of correlation 1, information measure of correlation 2 and normalized inverse difference moment [7].

3.2b Chebyshev moments: Vibha \& Rege [26] suggested use of modified Chebyshev moments for geometric 
transform invariant texture analysis. In order to achieve rotation and scale invariance, log-polar transform can be applied on the image before computation of the Chebyshev moments. The log-polar transform has an important property that it is rotation and scale invariant. Given an image $f(x, y)$ in Cartesian coordinates $(x, y)$ we can represent it in the polar log coordinate space $(\lambda, \theta)$ where $e^{\lambda}=\sqrt{ }\left(x^{2}+y^{2}\right)$ and $\theta=\tan ^{-1}\left(\frac{y}{x}\right) ; \theta €(-\pi, \pi)$.

The discrete ortho-normal Chebyshev moments $T_{p q}$ of order $p+q$, with size $N \times N$ for an image $f(x, y)$, are given as

$$
T_{p q}=\frac{1}{p(x, N) p(y, N)} \sum_{x=0}^{N-1} \sum_{y=0}^{N-1} \hat{t}_{p}(x) \hat{t}_{p}(y) f(x, y)
$$

where $x=y=0,1,2, \ldots,(N-1) ; \hat{t}_{p}(x)=\hat{t}_{p}(y)=p^{\text {th }}$ order Chebyshev moment.

First two terms and the recurrence relation for Chebyshev moments are given as

$$
\begin{gathered}
\hat{t}_{0}(x)=1 \\
\hat{t}_{1}(x)=\frac{(2 x-N+1)}{N} \\
\hat{t}_{p}(x)=\frac{(2 p-1) t_{1}(x) t_{p-1}(x)-(p-1)\left(1-\frac{p-1}{N^{2}}\right) t_{p-2}(x)}{p}, \text { if } p \geq 2 .
\end{gathered}
$$

Squared normalization factor can be written as

$$
p(x, N)=p(y, N)=\frac{N\left(1-\frac{1}{N^{2}}\right)\left(1-\frac{2^{2}}{N^{2}}\right) \cdots\left(1-\frac{n^{2}}{N^{2}}\right)}{2 n+1}
$$

where $n=0,1,2 \ldots(N-1)$.
In the present work, local neighborhood of size $8 \times 8$ and $16 \times 16$ is selected around the center of the ROI. Selected area is median filtered and transformed using logpolar transformation. Chebyshev moments of various orders are computed. By analyzing Chebyshev moment values for normal and abnormal ROIs, it is found that only second order Chebyshev moment $\mathrm{T}_{11}$ can differentiate well between normal and abnormal ROIs. Hence moment $\mathrm{T}_{11}$ is computed for $8 \times 8$ and $16 \times 16$ sub-blocks and used as local features.

\subsection{Global feature extraction}

Figure 5 illustrates the technique used for global feature extraction. The features are classified into three sets. First set is derived from original ROI in which given ROI is transformed using rectangular to polar transformation and then five Laws texture energy measures namely L1, L2, L3, L4 and L5 are computed. Second set of features is derived from Gabor filtered ROI where given ROI is preprocessed and passed through Gabor filter banks and transformed to polar system to give five Laws texture energy measures namely G1, G2, G3, G4, and G5. One more feature, G6 is derived from the two Gabor filtered Laws texture features, by taking the product of first and last Laws texture measures. Third set of features has two features D1 and D2 where D1 is a fractal dimension of original ROI and D2 is the fractal dimension of preprocessed ROI. Thus for every ROI, a global feature vector of size 13 is determined.

3.3a Gabor filtering: Gabor filters are useful in order to capture oriented texture patterns. The real Gabor filter kernel which is able to capture features at orientation of $-\pi /$ 2 is given by

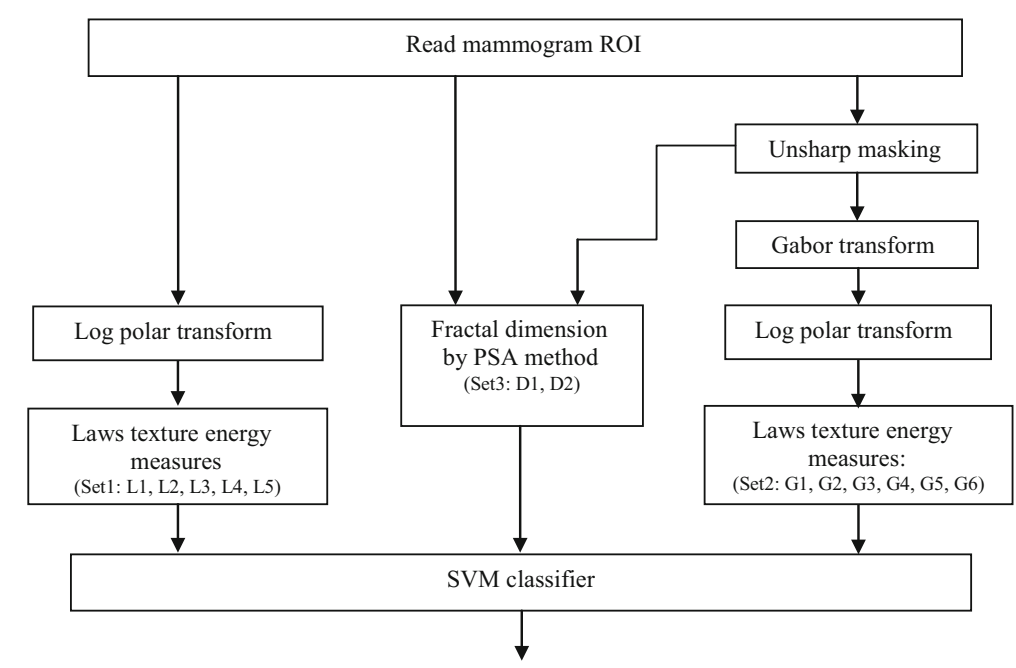

Figure 5. Global feature extraction for ROI. 


$$
g(x, y)=\frac{1}{2 \pi \sigma_{x} \sigma_{y}} \exp \left[-\frac{1}{2}\left(\frac{x^{2}}{\sigma_{x}^{2}}+\frac{y^{2}}{\sigma_{y}^{2}}\right)\right] \cos (2 \pi f x) .
$$

The parameters $\sigma_{x}, \sigma_{y}$ and $f$ represent standard deviation in the $X$ direction, standard deviation in the $Y$ direction and frequency of the modulating sinusoid respectively. This kernel is rotated to obtain kernels at other angles. Parameter values are derived by taking into account dimension of the structures to be detected. Physical dimension of the structures to be detected is $800 \mu \mathrm{m}$ and pixel size is $200 \mu \mathrm{m}$ in the MIAS database. Therefore, the full width, $w$ of Gaussian function at half maximum width is considered as four pixels for determining the values of $\sigma_{x}, \sigma_{y}$ and $f$. Selected values for $f, \sigma_{x}$ and $\sigma_{y}$ are $1 / w, w /$ (2.35) and $l . \sigma_{x}$ respectively for elongation in $Y$ direction, $l$ $=8$.

Gabor kernel as given in Eq. (8) is rotated at angles spaced evenly over the range $[-\pi / 2, \pi / 2]$ using the coordinate transformation. For each ROI, 180 Gabor filtered images are obtained by using 180 Gabor kernels. For every pixel in the ROI, Gabor magnitude responses are compared and the maximum of all is selected. Abnormal ROI and the

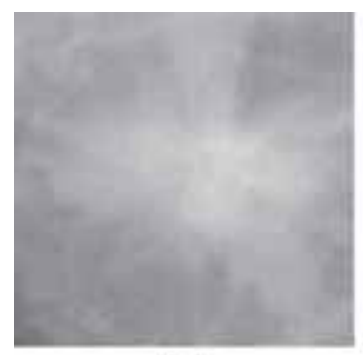

(a)

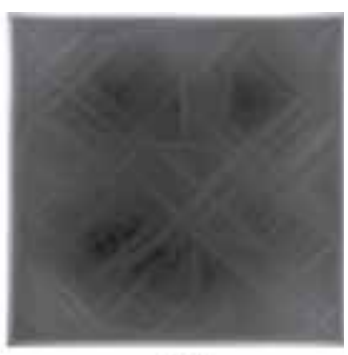

(b)
Figure 6. (a) Abnormal ROI; (b) magnitude response of Gabor filtered ROI. magnitude response of Gabor filtered preprocessed ROI are as shown in figure 6 .

3.3b Rectangular to polar transformation: The rectangular ROIs and their Gabor magnitude responses of the same size were transformed to the respective polar coordinates. The input ROI in $(x, y)$ coordinate system is of size $128 \times 128$. It is transformed to $(r, \theta)$ plane using rectangular to polar conversion. The origin of the image in $x-y$ plane is assumed to be at the center of the image. As ROI size is $128 \times 128$, the radial distance $r$ varies from 1 to 63 and $\theta$ varies from $0^{\circ}$ to $359^{\circ}$. Bilinear interpolation is used to interpolate between points which are not exactly in the image. This geometric transformation converts spiculated patterns into ripple or wave patterns which can be enhanced by Laws convolution masks. The results of rectangular to polar conversion of original ROI and Gabor filtered ROI are as shown in figure 7(a)-(b) and (c)-(d) respectively.

3.3c Laws texture energy measures: Laws developed a texture energy approach to measure the amount of variation within a fixed size window [19]. A set of five $5 \times 5$ masks is used to compute texture energy by convolving the mask with the given image. Laws masks are useful to emphasize specific structural patterns like level, edge, spot, ripple and wave pattern. Hence, Laws' texture measures could be used to generate useful features related to the interesting tissue structures like spiculations, and node-like patterns of architectural distortion.

Laws defined 1-D operators of length five pixels, such as


1]. The 2-D convolution masks of size $5 \times 5$ pixels are obtained by computing outer products of the pair of vectors. L5, R5, W5 are useful to emphasize center-weighted local average $\left(\mathrm{L} 5 \mathrm{~L} 5=\mathrm{L} 5^{\mathrm{T}} \mathrm{L} 5\right)$, ripples $\left(\mathrm{R} 5 \mathrm{R} 5=\mathrm{R} 5^{\mathrm{T}} \mathrm{R} 5\right)$ and wave patterns (W5W5 $\left.=\mathrm{W}^{\mathrm{T}} \mathrm{W} 5\right)$, respectively. Laws' L5L5, R5R5, W5W5, rotated R5R5 and rotated W5W5

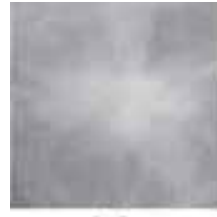

(a)

(b)

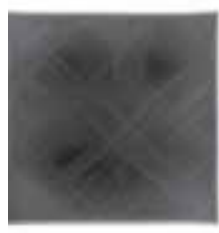

(c)

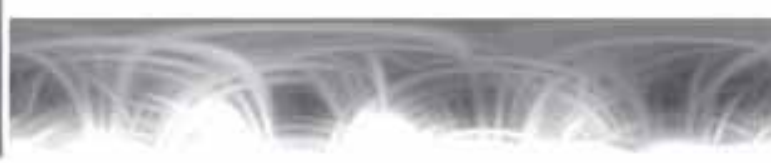

(d)

Figure 7. (a) Original ROI; (b) rectangular to polar conversion of (a); (c) magnitude response of Gabor filtered ROI; (d) rectangular to polar conversion of $(\mathbf{c})$. 

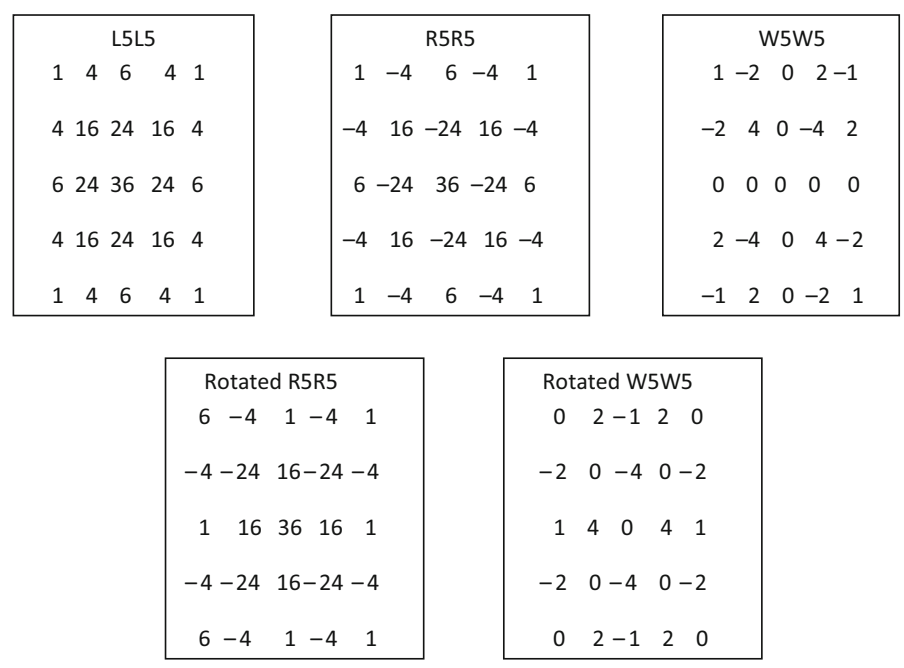

Figure 8. Laws' texture energy masks.

masks are shown in figure 8. The rectangular ROIs and their Gabor magnitude responses of the same size were transformed to the polar coordinates. The transformed ROIs and the transformed Gabor magnitude responses were convolved with Laws $5 \times 5$ convolution masks designed for the detection of waves (W5W5), ripples (R5R5), and the center-weighted local average (L5L5). In addition, two other masks, W5W5 rotated by 45 and R5R5 rotated by 45, were used. Following the application of the selected filters, texture energy measures were derived from each of the filtered images by computing the average of the squared values in a $15 \times 15$ sliding window. Finally, the sum of each of the energy measures normalized by the area of the transformed image was used to derive five features from the transformed ROIs and five from the transformed Gabor magnitude responses. One more feature is derived for the transformed Gabor magnitude responses by taking product of first and last Laws texture features.

3.3d Fractal dimension by power spectral analysis (PSA): The fractal property of self-similarity is evident in many biomedical systems and entities. In mammography, a few studies [21] have suggested that breast tissue patterns could appear as fractal objects. The presence of abnormality disrupts the self-similarity properties, and thereby changes the fractal dimension (FD) of breast parenchyma. As a result, fractal dimension can be used as a discriminating feature to distinguish between the normal patterns and abnormal patterns. Following steps are used to estimate the fractal dimension:

- Obtain the 2-dimensional (2-D) Fourier power spectrum of the given ROI.

- Integrate the 2-D power spectrum as a function of frequency from the zero-frequency point over the range in angle. This step converts 2-D power spectrum to 1-D spectrum and enhances the spectral characteristics of periodic or spiculated texture.

- Exclude points from the spectrum in the selected lowfrequency region in order to remove the effects of the low-frequency components. Overall appearance of the image and the large structures present in the image represent low frequency components which are not of interest.

- Exclude points from the spectrum in the selected highfrequency region to avoid the effects of high-frequency noise.

- Apply linear regression to the selected frequency range of the 1-D spectrum on a log-log scale.

- Estimate the slope $\beta$ of the fitted line.

- Determine the fractal dimension using $F D=(8-\beta) /$ 2.

Fractal dimensions of original ROI and sharpened ROI are computed using above steps and used as two additional global features for classifying abnormal and normal ROIs.

\subsection{Classification using SVM classifier}

Twenty-one local features and 13 global features are combined to form a feature vector for each ROI. A set of feature vectors is used to train SVM classifier for discriminating normal and abnormal regions. Main aim of SVM learning procedure is to minimize the bound on the generalization error, i.e. error made by the learning machine on data unseen during training rather than minimizing the mean square error over the data set. As a result, this leads to good generalization and an SVM tends to perform well when applied to data outside the training set. 
The key attribute of SVMs is to map the data into the feature space where a hyperplane (decision boundary) separating the classes may exist. This mapping is achieved via the use of kernels, which return the scalar product in the feature space by performing calculations in the data space. The simplest is the linear SVM trained to classify linearly separable data. The distance between the two parallel hyperplanes on which the support vectors for the respective classes fall is called the margin. SVM finds a decision boundary that maximizes the margin. For non-linear classification, kernels are used to map the data into a higher dimensional feature space in which a linear separating hyperplane can be found.

Here Artificial neural network (ANN) is not used for classification as it is not very good in generalization. During ANN training of network, in verification phase, ANN gives very good accuracy but when the trained network is used for classification of test data set, it gives poor performance. SVM classifier proved superior to the ANN in generalization.

\section{Results and discussion}

The proposed algorithm has been tested on a standard desktop machine by using Matlab 7.14.0.739. We considered five types of benign and malignant abnormalities namely, microcalcifications, circumscribed masses, spiculated masses, architectural distortions and ill-defined (miscellaneous) abnormality. The database is divided into two subsets. One set is used to train the SVM classifier and the other is used to test the SVM classifier. The various kernel functions tested for the SVM classifier are radial basis function (sigma value 0.8-2.5), polynomial (order 2-7) and linear function. Best results are obtained by training the SVM classifier by using radial basis function with $\sigma$ value of 2.1.

Performance is measured in terms of sensitivity, specificity and accuracy. Sensitivity indicates percentage of correctly classified abnormal samples; specificity gives percentage of correctly classified normal samples whereas accuracy indicates percentage of correctly classified samples (normal as well as abnormal) out of total samples.

Performance of classification is tested for the three cases. SVM classifier is trained using only global features, using only local features and combined use of local and global features. The sensitivity, specificity and accuracy of the proposed system, for the three cases are given in table 1 . From the results it is clear that when classification is done using only global features, specificity is $86.92 \%$ indicating large number of false positives. Combined use of local and global features has improved specificity to $93.46 \%$ and hence improved the accuracy of classification to $93.17 \%$ from $88.75 \%$.
Table 1. Performance measures.

\begin{tabular}{lccc}
\hline & \multicolumn{3}{c}{ Testing set } \\
\cline { 2 - 4 } Features & Sensitivity (\%) & specificity (\%) & Accuracy (\%) \\
\hline Local & 81.25 & 84.97 & 83.53 \\
Global & 91.67 & 86.92 & 88.75 \\
Global + local & 92.71 & 93.46 & 93.17 \\
\hline
\end{tabular}

Texture of the abnormality in the mammogram is different than that of the normal tissue. Usually contrast of the masses is lower than the contrast of normal region. Hence texture features like contrast, cluster shade, sum of squares, sum average and other are useful for differentiating between normal and abnormal tissues. In the proposed work, all the features local as well as global are ranked in descending order of the importance of the individual feature to distinguish between normal and abnormal patterns. Then SVM is trained and tested using selected features up to particular rank. It is observed that by addition of selected features, accuracy of classification is improved. For example with first six features as per the rank, (product of first and fifth Gabor feature, fractal dimension of ROI, fifth and first Gabor feature, Chebyshev moment $\mathrm{T}_{11}$ over neighborhood of $8 \times 8$ and autocorrelation of GLCM) accuracy was $87.55 \%$ and with first 22 features as per the rank, accuracy was $89.56 \%$. When all 34 features were used accuracy was increased to $93.17 \%$. Thus each and every feature is contributing in the classification.

Table 2 shows comparison of the results of the proposed approach with the reported in the literature on the qualitative basis. In the context of the results of the reported work, the results of the proposed method are comparable and encouraging. Though the accuracy obtained in the proposed method is slightly less than the accuracy reported by Shanthi \& Murali Bhaskaran [9], proposed method is capable of detecting five types of abnormalities namely microcalcifications (CALC), circumscribed masses (MASS), spiculated masses (SPIC) architectural distortions (ARCH) and ill-defined or miscellaneous masses (MISC). In the work by Phadke \& Rege [8], sensitivity is higher than the sensitivity of the proposed system. As the former technique classifies only malignant samples whereas proposed system classifies both malignant and benign abnormalities from normal samples. If sensitivity is poor, it means abnormality goes undetected while a low value of specificity needs the normal patient to undergo biopsy. Thus, sensitivity and specificity both are expected to be high. Accuracy has priority for testing the performance of mammogram classification system. The accuracy of proposed system is greater than the system by Phadke and Rege [8]. 
Table 2. Comparison of the proposed algorithm with results reported in the literature.

\begin{tabular}{|c|c|c|c|c|}
\hline Author & $\begin{array}{l}\text { Sign of breast } \\
\text { cancer }\end{array}$ & Database & Method & Performance \\
\hline $\begin{array}{l}\text { Phadke \& Rege } \\
\text { (proposed } \\
\text { method) }\end{array}$ & $\begin{array}{l}\text { CALC, MASS, } \\
\text { ARCH, SPIC, } \\
\text { MISC }\end{array}$ & MIAS & $\begin{array}{l}\text { Global features like Laws texture energy measures, Gabor-laws } \\
\text { features, fractal dimension and local features like chebyshev } \\
\text { moment and } 19 \text { GLCM features, classification using SVM }\end{array}$ & $\begin{array}{c}\text { Accuracy } 93.17 \% \text {; } \\
\text { sensitivity } \\
92.71 \%\end{array}$ \\
\hline $\begin{array}{l}\text { Shanthi \& } \\
\text { Murali } \\
\text { Bhaskaran [9] }\end{array}$ & $\begin{array}{c}\text { CALC, MASS, } \\
\text { ARCH }\end{array}$ & MIAS & $\begin{array}{c}\text { Feature set based on Gabor filters, fractal analysis, multiscale } \\
\text { surrounding region dependence method (MSRDM) self- } \\
\text { adaptive resource allocation network (SRAN) classifier }\end{array}$ & Accuracy $98.44 \%$ \\
\hline $\begin{array}{l}\text { Phadke \& Rege } \\
\text { [8] }\end{array}$ & $\begin{array}{l}\text { CALC, MASS, } \\
\text { ARCH, SPIC, } \\
\text { MISC }\end{array}$ & MIAS & Use of PCA and ICA features and classification using ANN & $\begin{array}{c}\text { Accuracy } 87.81 \% \text {; } \\
\text { sensitivity } \\
94.72 \%\end{array}$ \\
\hline Oliver et al [16] & CALC & $\begin{array}{l}\text { MIAS, } \\
\text { Real } \\
\text { Dataset }\end{array}$ & $\begin{array}{l}\text { Knowledge based approach for classification using local } \\
\text { features extracted from bank of filters }\end{array}$ & $\begin{array}{l}\text { Sensitivity } 80 \% \text { at } \\
1 \mathrm{FP} / \mathrm{image}\end{array}$ \\
\hline Banik et al [19] & $\mathrm{ARCH}$ & $\begin{array}{l}\text { Real } \\
\text { Dataset }\end{array}$ & $\begin{array}{l}\text { Linear phase portrait analysis for detection of potential sites of } \\
\text { architectural distortion and use of fractal dimension, angular } \\
\text { spread of power and texture features }\end{array}$ & $\begin{array}{l}\text { Sensitivity } 90 \% \text { at } \\
8.1 \mathrm{FP} / \text { image }\end{array}$ \\
\hline $\begin{array}{l}\text { Biswas \& Dipti } \\
\quad[18]\end{array}$ & $\mathrm{ARCH}$ & $\begin{array}{l}\text { MIAS, } \\
\text { DDSM }\end{array}$ & $\begin{array}{l}\text { Multiscale texture modeling approach with Gaussian mixture } \\
\text { model }\end{array}$ & $\begin{array}{r}\text { Sensitivity } 80.7 \% \\
\text { at } 3.9 \mathrm{FP} / \mathrm{image}\end{array}$ \\
\hline $\begin{array}{l}\text { Karahaliou et al } \\
\text { [11] }\end{array}$ & CALC & DDSM & $\begin{array}{l}\text { Texture features extracted from the tissue surrounding the } \\
\text { abnormality and classification using KNN classifier }\end{array}$ & $\begin{array}{c}\text { Sensitivity } \\
94.00 \% \\
\text { specificity } \\
80.00 \%\end{array}$ \\
\hline
\end{tabular}

\section{Conclusion}

In this work, a method is developed for classification of normal and abnormal ROI of breast tissues from mammograms using fusion of local and global features. The local features used are Chebyshev moment $T_{11}$ for two sizes of neighborhood and 19 GLCM features whereas for global features, five Laws texture energy measures of ROI, six Laws texture energy measures of Gabor magnitude response of ROI and fractal dimension are used. Proposed system gives promising results in classification of normal and abnormal patterns by using SVM classifier with radial basis function kernel. Accuracy of classification of the proposed system is $93.17 \%$. From the results, it can be concluded that fusion of local and global features leads to improvement in sensitivity, specificity and accuracy.

Combined use of PCA-ICA features with features used in the proposed method can be tested for classification of samples into three classes: normal, benign and malignant. New features can be added to the proposed system in order to further improve the overall performance of the system.

\section{Acknowledgements}

This work was funded by Board of College and University Development, University of Pune. Authors are grateful to Board of College and University Development for funding the project. The authors are also thankful to oncologists Dr. Shekhar Kulkarni and Dr. Aparna Atre for their valuable inputs. Authors would like to thank the reviewers for giving guidelines for improvement in the quality of the manuscript.

\section{References}

[1] Datta Karabi, Choudhary Maitrayee, Guha Subhas and Biswas Jaidip 2012 Breast cancer scenario in a Regional Cancer Centre in Eastern India over eight years- still a major public health problem. Asian Pacific J. Cancer Prevent. 13: 809-813

[2] American College of Radiology (ACR) 1998 Illustrated breast imaging reporting and data system (BI-RADS), 3rd edition. American College of Radiology, Reston, VA

[3] Eberl Margaret M, Fox Chester H, Edge Stephen B, Carter Cathleen A and Mahoney Martin C 2006 BI-RADS classification for management of abnormal mammograms. J. Am. Board Fam. Med. 19(219): 161-164

[4] Vibha S V and Rege P P 2007 Malignancy texture classification in digital mammograms based on Chebyshev moments and $\log$ polar transformation. ICGST-BIME J. 7(1): 29-35

[5] Rashed Essam A, Ismail A Ismail and Zaki Sherif I 2007 Multiresolution mammogram analysis in multilevel decomposition. Elsevier Pattern Recognit. Lett. 28: 286-292

[6] Tirtajaya Andy and Santika Diaz D 2010 Classification of microcalcification using dual-tree complex wavelet transform and support vector machine. In: Second International Conference on Advances in Computing, Control, and Telecommunication Technologies, IEEE computer society, 164-166

[7] Nithya R and Santhi B 2011 Mammogram classification using maximum difference feature selection method. $J$. Theoret. Appl. Inform. Technol. 33(2): 197-204 
[8] Phadke A C and Rege P P 2014 Comparison of SVM \& ANN Classifier for Mammogram classification using ICA features. WIT Trans. Inform. Commun. Technol. 49: 497-504

[9] Shanthi S and Murali Bhaskaran V 2014 A novel approach for classification of abnormalities in digitized mammograms. Sadhana, Indian Acad. Sci. 39(5): 1141-1150

[10] Ted C Wang and Nicolaos B Karayiannis 1998 Detection of microcalcifications in digital mammograms using wavelets. IEEE Trans. Med. Imaging 17(4): 498-509

[11] Karahaliou A, Skiadopoulos S, Boniatis I, Sakellaropoulos P, Likaki E, Panayiotakis G and Costaridou L 2007 Texture analysis of tissue surrounding microcalcifications on mammograms for breast cancer diagnosis. Br. J. Radiol. 80: 648-656

[12] Alarcon-Aquino V, Starostenko O, Ramirez-Cortes J M, Rosas-Romero R, Rodriguez-Asomoza J, Paz-Luna O J and Vazquez-Muñoz K 2009 Detection of microcalcifications in digital mammograms using the dual-tree complex wavelet transform. Eng. Int. Syst., CRL Publishing Ltd, 49-63

[13] Balakumaran T, Vennila L and Shankar Gowri 2010 Detection of microcalcification in mammograms using wavelet transform and fuzzy shell clustering. Int. J. Comput. Sci. Inform. Security 7(1): 657-660

[14] Leena Jasmine J S, Govardhan A and Baskaran S 2010 Classification of microcalcification in mammograms using nonsubsampled contourlet transform and neural network. Eur. J. Sci. Res. 46(4): 531-539

[15] Paradkar Sarvesh and Pande S S 2011 Intelligent detection of microcalcification from digitized mammograms. Sadhana, Indian Acad. Sci. 36(1): 125-139

[16] Oliver Arnau, Torrent Albert, Xavier Llado, Meritxell Tortajada, Lidia Tortajada, Melcior Sentis, Jordi Freixenet and Reyer Zwiggelaar 2012 Automatic microcalcification and cluster detection for digital and digitized mammograms. Elsevier Knowl. Based Syst. 28: 68-75

[17] Sampat Mehul P and Bovik Alan C 2003 Detection of spiculated lesions in mammograms. In: Proceedings of the 25 Annual International Conference of the IEEE EMBS Cancun, Mexico 17-21 September 2003, 810-813
[18] Biswas Sujay and Mukherjee Dipti 2011 Recognizing architectural distortion in mammogram: A multiscale texture modeling approach with GMM. IEEE Trans. Biomed. Eng. 58(7): 2023-2030

[19] Banik Shantanu, Rangayyan Rangaraj M and Leo J E 2011 Detection of architectural distortion in prior mammograms. IEEE Trans. Med. Imaging 30(2): 279-294

[20] Phadke A C and Rege P P 2013 Classification of architectural distortion from other abnormalities in mammograms. Int. J. Appl. Innov. Eng. Manag. 2(2): 42-48

[21] Rangayyan R M and Nguyen T M 2007 Fractal analysis of contours of breast masses in mammograms. J. Digit. Imaging 20(3): 223-237

[22] Apffel Louis, Palma Giovanni, Muller Serge and Isabelle Bloch 2010 Fuzzy segmentation of masses in digital breast tomosynthesis images based on dynamic programming. IMAGAPP 2010 - International Conference on Imaging Theory and Applications, 7-13

[23] Arianna Mencattini, Giulia Rabottino, Marcello Salmeri, Roberto Lojacono and Emanuele Colini 2011 Automatic breast masses boundary extraction in digital mammography using spatial fuzzy c-means clustering and active contour models. IEEE Conference on Medical Imaging, November 8, 2011, 978-1-4244-9338

[24] Suckling J, Astley S, Betal D, Cerneaz N, Dance D R and Kok S L 1994 The mammographic image analysis society digital mammogram database. International Congress Series 1069. Excerpta Medica 375-378. Available at http://peipa. essex.ac.uk/info/mias.html

[25] Khuzi Mohd, Besar R, Zaki W M D Wan and Ahmad N N 2009 Identification of masses in digital mammogram using gray level co-occurrence matrices. Biomed. Imaging Intervention J. 5(3): e17

[26] Vibha S V and Rege P P 2009 Geometric transform invariant texture analysis with modified Chebyshev moments based algorithm. Int. J. Image Graphics, World Scientific Publishing Company 9(4): 1-16 